\title{
Crystal forms in pharmaceutical applications: Olanzapine, a gift to crystal chemistry that keeps on giving
}

\author{
Susan Reutzel-Edens ${ }^{1}$, Rajni Bhardwaj ${ }^{2}$ \\ ${ }^{1}$ Cambridge Crystallographic Data Centre, Cambridge, United Kingdom; \\ ${ }^{2}$ AbbVie, North Chicago, IL USA; \\ sreutzel-edens@ccdc.cam.ac.uk
}

We review the efforts of many scientists around the world to discover and structurally characterize olanzapine crystal forms, clearing up inconsistencies in the scientific and patent literature and highlighting the challenges in identifying new forms amidst $60+$ known polymorphs and solvates.[1] Owing to its remarkable solid-state chemistry, olanzapine has emerged over the last three decades as a popular tool compound for developing new experimental and computational methods for enhanced molecular level understanding of solid-state structure, form diversity and crystallization outcomes. This presentation highlights the role of olanzapine in advancing the fundamental level understanding of crystal forms, interactions within crystal structures, and growth units in molecular crystallization, and in influencing the way in which drugs are developed to this day.

[1] Reutzel-Edens, S.M., Bhardwaj, R. M. IUCrJ (2020). 7, 955-964 https://doi.org/10.1107/S2052252520012683

Keywords: polymorphism, crystallization, hydrate, solid state, pharmaceutical 